\title{
Corrections
}

\section{Correction: Klein-Flügge, Barron, et al., Segregated Encoding of Reward-Identity and Stimulus-Reward Associations in Human Orbitofrontal Cortex}

In the article "Segregated Encoding of Reward-Identity and Stimulus-Reward Associations in Human Orbitofrontal Cortex" by Miriam Cornelia Klein-Flügge, Helen Catharine Barron, Kay Henning Brodersen, Raymond J. Dolan, and Timothy Edward John Behrens, which appeared on pages 3202-3211 of the February 13, 2013 issue, we misstated the echo time (TE) of our scan sequence as $70 \mathrm{~ms}$ when it was actually $30 \mathrm{~ms}$. The corrected sentence on page 3204, first column, last paragraph reads as follows: “A special sequence was used to minimize signal drop out in the OFC region (Weiskopf et al., 2006) and included an echo time (TE) of $30 \mathrm{~ms}$, a tilt of $30^{\circ}$ relative to the rostro-caudal axis, and a local $z$-shim with a moment of $0.4 \mathrm{mT} / \mathrm{m}$ ms applied to the OFC region.” This error does not affect any of the conclusions or interpretations in the paper.

DOI: 10.1523/JNEUROSCI.2000-16.2016 